\title{
Reporte de los primeros 50 pacientes con COVID-19 en ventilación mecánica hospitalizados en la Unidad de Cuidados Intensivos del Complejo Asistencial Dr. Sótero del Río
}

\author{
TOMÁS LAHERA ${ }^{1}$, CAROLINA RUIZ $^{1,2}$, ANDRÉS AQUEVEDO $^{1,2}$, \\ PETRE COTORAS $^{3}$, JAVIER URIBEA $^{A}$, CLAUDIO MONTENEGRO ${ }^{4}$
}

\section{Features of 50 patients with SARS-CoV2 admitted to an intensive care unit and requiring mechanical ventilation}

Background: Since March, Chile experienced an exponential increase in SARS-CoV2 cases, which led to an almost full occupancy of the intensive care units (ICU). Aim: To characterize patients with SARS-CoV2 disease who required hospitalization in the ICU and invasive mechanical ventilation (IMV) in our hospital. Material and Methods: A prospective observational study was performed, which included the first 50 patients, aged $54 \pm 13$ years ( $58 \%$ men), with SARS-CoV2 disease, with ICU and IMV requirements between March 23 and June 2, 2020. Demographics, comorbidities, symptoms, laboratory and imaging, therapies performed and IMV characteristics were registered. The most relevant outcomes observed were lethality, number of days in the ICU and connection to an IMV. Results: Ninety percent of patients were overweight or obese, $46 \%$ had hypertension and many had diabetes mellitus. They had elevated inflammatory parameters and typical patterns of COVID-19 in computed tomography. Most of the patients got protective lung ventilation with a high rate of use of neuromuscular blockade (NMB) and prone position. Antibiotics, hydroxychloroquine, and lopinavir/ritonavir were administered according to the protocol of the institution. Lethality was 16\% (8 cases) at the end of this study. Conclusions: The information obtained in this study provides characteristics and early outcomes of hospitalized patients with confirmed COVID-19 and $I M V$, admitted to the ICU of our center.

(Rev Med Chile 2020; 148: 1725-1733)

Key words: COVID-19; Critical Care; Epidemiology; Respiratory Insufficiency.

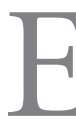
1 primer caso confirmado en Chile de enfermedad por coronavirus o COVID-19 (Coronavirus-disease) fue diagnosticado el 3 de marzo de 2020. Esta enfermedad es causada por un nuevo beta-coronavirus, que actualmente se conoce como SARS-CoV2 (severe acute res-
'Unidad de Cuidados Intensivos, Complejo Asistencial Dr. Sótero del Río. Santiago, Chile. ${ }^{2}$ Departamento de Medicina Intensiva, Escuela de Medicina, Facultad de Medicina, Pontificia Universidad Católica de Chile.

Santiago, Chile.

${ }^{3}$ Departamento de Medicina Interna. Escuela de Medicina, Facultad de Medicina, Pontificia Universidad Católica de Chile.

Santiago, Chile.

${ }^{4}$ Servicio de Imagenología,

Complejo Asistencial Dr. Sótero del Río. Santiago, Chile.

aResidente de Medicina Interna. Escuela de Medicina, Facultad de Medicina, Pontificia Universidad Católica de Chile. Santiago, Chile.

Los autores declaran no tener conflictos de interés.

Trabajo no recibió financiamiento.

Recibido el 29 de junio de 2020, aceptado el 10 de noviembre de 2020.

\section{Correspondencia a:} Dra. Carolina Ruiz Av. Concha y Toro 3459, Puente Alto, Santiago, Chile. ccruiz@uc.cl piratory syndrome Coronavirus 2). Entre abril y junio ha habido un aumento exponencial de los casos de COVID-19 en nuestro país, con un total de 275.648 casos al 22 de junio de $2020^{1}$. Esto ha generado una gran demanda para las unidades de cuidados intensivos (UCI), ya que la proporción 
de pacientes que ingresa a estas unidades se ha reportado entre 6 y $26 \%$ de todos los hospitalizados $^{2-4}$.

Hasta la fecha existe poca información de las características de los pacientes hospitalizados por esta patología en UCI en Chile. En un estudio realizado en Nueva York, 14,2\% de los pacientes requirió ingreso a UCI, con una letalidad elevada para aquellos que recibieron ventilación mecánica invasiva $(\mathrm{VMI})^{5}$. No obstante, existen marcadas diferencias entre la población de Estados Unidos y Chile, tanto desde el punto de vista demográfico, presencia de comorbilidades y características de los sistemas sanitarios.

El objetivo de este estudio fue describir las características, tratamientos y desenlaces clínicos de los primeros 50 pacientes que ingresaron a la UCI de adultos del Complejo Asistencial Dr. Sótero del Río por COVID-19 (CASR) y necesitaron VMI.

\section{Pacientes y Método}

Se realizó un estudio observacional y prospectivo en la UCI del CASR, que es un hospital público de alta complejidad de Santiago de Chile. Este centro cuenta con una población asignada de 1.495.821 habitantes ${ }^{6}$. La UCI del CASR es una unidad que cuenta con todos los soportes avanzados, incluido ECMO (extracorporeal membrane oxygenation), junto con un equipo de profesionales (médicos, enfermeras, kinesiólogos, etc.) certificados en medicina intensiva. Esta unidad habitualmente tenía 18 camas, aumentando a 42 a mediados de abril de 2020, mediante la reconversión de camas de intermedio en UCI, debido a la pandemia de COVID-19. El estudio fue aprobado por el Comité de Ética institucional (Comité de Ética del Servicio de Salud Metropolitano Sur Oriente), que determinó que no era necesario consentimiento informado.

Se planificó incluir a los primeros 50 pacientes adultos (mayores de 17 años) que ingresaron a UCI por una falla respiratoria aguda por COVID-19 y que requirieron conexión a VMI. El reclutamiento se inició el 23 de marzo de 2020, día en que ingresó el primer paciente. Los pacientes fueron reclutados de manera consecutiva y el registro de variables se realizó hasta 7 días tras el enrolamiento del último paciente. Se consideró infección confirmada con al menos un examen de reacción de polimerasa en cadena (PCR) para
SARS-CoV2 positivo. En los casos inicialmente negativos, si la sospecha clínica era alta, se repitió el test tras $72 \mathrm{~h}$.

Los datos, recolectados del sistema informático del CASR (PULSO $\left.{ }^{\circledR}\right)$, incluyeron: información demográfica, comorbilidades, variables clínicas, parámetros de VMI, resultados de exámenes, tratamientos e infecciones sobreagregadas. Se describieron como principales resultados clínicos: letalidad, días de UCI y días de VMI.

El tratamiento del COVID-19 fue guiado por el protocolo del hospital del 7 de abril $^{7}$, que recomendaba, ante la presencia de infección respiratoria baja con signos de gravedad por ATS/IDSA ${ }^{8}$, el uso precoz de hidroxicloroquina, lopinavir/ ritonavir y antibióticos (betalactámicos y azitromicina). Además consideraba la administración de corticoides frente a: pacientes con PCR (proteína $\mathrm{C}$ reactiva) y/o LDH mayor(es) a 3 veces el valor basal, pacientes con niveles de ferritina mayores a $2.000 \mathrm{ng} / \mathrm{mL}$ o pacientes con dímero D (DD) mayor a $1.000 \mathrm{ng} / \mathrm{mL}$. Previo a los corticoides había que descartada sobreinfección. Por último, se podía utilizar Tocilizumab frente al desarrollo de falla multiorgánica y ausencia de respuesta a corticoides.

Se uso VMI protectora, que es el estándar de manejo en nuestra UCI, programando un volumen corriente (VC) entre 6-7 ml/kg de peso ideal, para metas de presión meseta (Pplt) y presión de distensión (Pplt-presión positiva del final de espiración, PEEP) menores a $30 \mathrm{mmHg}$ y $15 \mathrm{mmHg}$ respectivamente; en caso de no lograr estas metas, se podía bajar el VC. Se utilizó sedación profunda, agregando bloqueo neuro muscular (BNM) si la $\mathrm{PaFi}$ (presión parcial de oxígeno arterial/fracción inspirada de oxígeno) era menor a 150. Si la PaFi persistía menor a 150, según el protocolo del estudio PROSEVA' 9 , se indicaba prono.

El seguimiento de las variables clínicas, exámenes y parámetros de VMI se realizó durante los primeros 7 días en UCI, a excepción de los signos vitales, en que también se consideraron los valores al ingreso al servicio de urgencia (SU). Respecto a los exámenes, en las tomografías computadas (TC) de tórax se determinó específicamente la presencia de los 3 patrones (típico, indeterminado y atípico) asociados a COVID- $19^{10}$. El seguimiento de los tratamientos, sobreinfecciones, días de UCI, duración de la VMI y letalidad se prolongó hasta el egreso de UCI o cierre del estudio. 
Por ser un estudio descriptivo, las variables dicotómicas se describieron con porcentajes y las contínuas con promedio/desviación standard o mediana/rango intercuartil 25-75.

\section{Resultados}

Un total de 50 pacientes fueron incluidos, entre el 23 de marzo y 25 de mayo de 2020, ingresando la mayoría desde el SU. Cinco pacientes se trasladaron desde otros servicios, pues, habían ingresado por otras patologías, diagnosticándoles posteriormente COVID-19. Todos los pacientes tuvieron al menos 1 PCR de hisopado nasofaríngeo para SARS-CoV2 positiva. La edad promedio fue $54 \pm 13$ años y la mayoría de los pacientes fueron hombres (58\%). Las comorbilidades más frecuentes fueron la obesidad $(48 \%)$ y la hipertensión arterial (46\%). Solo 10\% de los pacientes eran normopesos (Figura 1). Los síntomas más frecuentes previos al ingreso fueron: disnea $(84 \%)$, tos $(72 \%)$ y fiebre $(58 \%)$. En la mayoría de los pacientes, el intervalo desde el inicio de los síntomas a la conexión a VMI fue 5-8 días. La Tabla 1 muestra las características basales.

El APACHEII (Acute Physiology And Chronic Health Evaluation II) y el score SOFA (Secuential-related Organ Failure Assessment) del día 1 en UCI fueron $20,7 \pm 10,5$ y 7,9 $\pm 4,3$ respectivamente. La mayoría de los pacientes no tuvieron

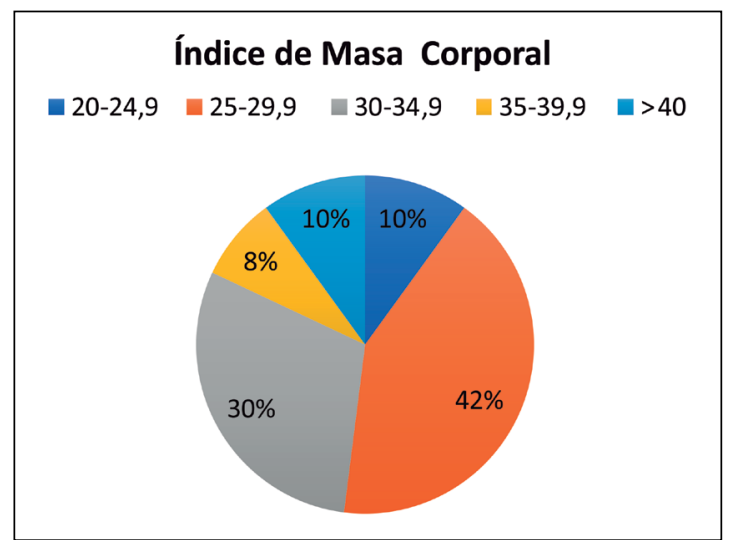

Figura 1. Estado nutricional de los pacientes por índice de masa corporal al ingreso a UCI. IMC calculado al ingreso de los pacientes a la UCI. Ningún paciente tenía IMC menor a 20. Abreviaturas: IMC, índice de masa corporal; UCI, unidad de cuidados intensivos.
Tabla 1. Características basales

\begin{tabular}{|c|c|c|}
\hline Variables & $\begin{array}{c}\text { N/ } \\
\text { Promedio }\end{array}$ & $\begin{array}{c}\text { Porcentaje/ } \\
\text { DE }\end{array}$ \\
\hline \multicolumn{3}{|l|}{ Sexo } \\
\hline - Hombre & 29 & $58 \%$ \\
\hline - Mujer & 21 & $42 \%$ \\
\hline \multicolumn{3}{|l|}{ Edad } \\
\hline - Promedio & 54 & \pm 13 \\
\hline$-18-40$ & 9 & $18 \%$ \\
\hline$-41-50$ & 11 & $22 \%$ \\
\hline$-51-60$ & 14 & $28 \%$ \\
\hline$-61-70$ & 11 & $22 \%$ \\
\hline$-71-80$ & 5 & $10 \%$ \\
\hline \multicolumn{3}{|l|}{ Comorbilidades } \\
\hline - HTA & 23 & $46 \%$ \\
\hline - Obesidad(a) & 24 & $48 \%$ \\
\hline - DM2 & 22 & $44 \%$ \\
\hline - Cardiopatías(b) & 5 & $10 \%$ \\
\hline - Enf. pulmonar ${ }^{(c)}$ & 3 & $6 \%$ \\
\hline - Inmunosupresión ${ }^{(d)}$ & 4 & $8 \%$ \\
\hline \multicolumn{3}{|l|}{ Síntomas } \\
\hline - Disnea & 42 & $84 \%$ \\
\hline - Tos & 36 & $72 \%$ \\
\hline - Fiebre & 29 & $58 \%$ \\
\hline - Mialgias & 17 & $34 \%$ \\
\hline - Odinofagia & 13 & $26 \%$ \\
\hline \multicolumn{3}{|l|}{ Días de evolución previo a VMI } \\
\hline - Promedio & 8 & $\pm 3,4$ \\
\hline $\begin{array}{l}-<5 \\
-5-8\end{array}$ & $\begin{array}{r}6 \\
24\end{array}$ & $12 \%$ \\
\hline $\begin{array}{l}-9-12 \\
-9\end{array}$ & 12 & $24 \%$ \\
\hline$->12$ & 8 & $16 \%$ \\
\hline \multicolumn{3}{|l|}{ Signos Vitales SU } \\
\hline - PAM (mm/Hg) & 92 & $\pm 17,4$ \\
\hline - FC (latidos/min) & 100 & $\pm 18,9$ \\
\hline - $\mathrm{T}^{\circ}>37,5^{\circ} \mathrm{C}$ (fiebre) & 15 & $30 \%$ \\
\hline - Sat. < 93\% con > 3lt $\mathrm{O}_{2}$ & 23 & $26 \%$ \\
\hline
\end{tabular}

Los promedios se informan con desviación estándar $( \pm)$. Abreviaturas: $\mathrm{N}$, número de pacientes o número de eventos; $\mathrm{DE}$, desviación estándar; HTA, hipertensión arterial; DM2, diabetes mellitus 2; enf., enfermedad; VMI, ventilación mecánica invasiva; SU, servicio de urgencia; PAM; presión arterial media; $\mathrm{FC}$, frecuencia cardíaca; $\mathrm{T}^{\circ}$, temperatura; Sat, saturometría de pulso; $\mathrm{O}_{2}$, oxígeno; ${ }^{(a)}$ Se considera obesidad con IMC > 29,9; (b)Cardiopatías: insuficiencia cardíaca y cardiopatía coronaria; (c)Enfermedades pulmonares: enfermedad pulmonar obstructiva crónica, asma y enfermedad pulmonar intersticial difusa; (d)Inmunosupresión: virus de inmunodeficiencia humana, quimioterapia citotóxica y pacientes que usan medicamentos inmunosupresores. 
compromiso hemodinámico, tanto en el SU, como durante el segumiento en UCI. Sin embargo, durante la estadía en UCI 50\% presentó elevación del lactato (Tabla 2). La droga vasoactiva más utilizada fue la noradrenalina, la que fue administrada en $58 \%$ de los pacientes en dosis bajas $(0,03$ a $0,1 \mathrm{ug} /$ $\mathrm{kg} / \mathrm{min}$ ), en el contexto de sedación y BNM. Aunque sólo $30 \%$ de los pacientes tuvieron fiebre en el SU, más del $60 \%$ presentó fiebre en UCI.

De los pacientes que ingresaron con síndrome de distrés respiratorio agudo (98\%), según la clasificación de Berlín ${ }^{11}$, 16\% eran severos $(\mathrm{PaFi}$ $<100$ ), $60 \%$ moderados (PaFi $100-200)$ y $22 \%$ leves (PaFi 200-300). La Figura 2 muestra la evolución de la PaFi. Se utilizó ventilación protectora, como se explicó previamente, siendo la ventilación controlada por volumen el modo más usado. El PEEP promedio durante el estudio fue $11 \pm 5,9$ cms $\mathrm{H}_{2} \mathrm{O}$. La mediana de duración de la VMI fue 16 (13-21) días. La Tabla 2 describe las variables más importantes relacionadas con el manejo ventilatorio.

Dentro de los exámenes de laboratorio, destaca el alto porcentaje de pacientes que presentaron leucocitosis $(80 \%)$, linfopenia $(88 \%)$ y elevación del DD (100\%); siendo el mayor valor encontrado $40.500 \mathrm{ng} / \mathrm{ml}$, en un paciente que tuvo un tromboembolismo pulmonar y falleció en UCI. El 100\% de los pacientes tuvieron elevación de la PCR, cuya mediana fue 52 veces el valor de corte. El resto de las variables de laboratorio están descritas en la Tabla 3. Todos los pacientes
Tabla 2. Manejo ventilatorio

\begin{tabular}{|c|c|c|}
\hline Variables & $\begin{array}{c}\text { N/ } \\
\text { Promedio }\end{array}$ & $\begin{array}{c}\text { Porcentaje/ } \\
\text { DE }\end{array}$ \\
\hline \multicolumn{3}{|l|}{ Presión meseta $\left(\mathrm{cms}_{2} \mathrm{O}\right.$ ) } \\
\hline - Día 1 & 21,5 & \pm 4 \\
\hline - Día 3 & 21,7 & $\pm 2,9$ \\
\hline - Día 7 & 21,1 & $\pm 3,5$ \\
\hline \multicolumn{3}{|c|}{ Presión distensión (cms H²O) } \\
\hline - Día 1 & 10,6 & $\pm 5,5$ \\
\hline - Día 3 & 11,8 & \pm 6 \\
\hline - Día 7 & 11,2 & $\pm 6,1$ \\
\hline \multicolumn{3}{|l|}{$\mathrm{PaFi}$} \\
\hline - Día 1 & 171 & $\pm 69,26$ \\
\hline - Día 3 & 229 & $\pm 72,83$ \\
\hline - Día 7 & 223 & $\pm 72,96$ \\
\hline \multicolumn{3}{|l|}{$\mathrm{pCO}_{2}(\mathrm{~mm} / \mathrm{Hg})$} \\
\hline - Día 1 & 42,9 & $\pm 22,2$ \\
\hline - Dís 3 & 42,0 & $\pm 21,7$ \\
\hline - Día 7 & 40,8 & $\pm 20,8$ \\
\hline$-\mathrm{pCO}_{2}>50$ & 4 & $8,3 \%$ \\
\hline Bloqueo Neuromuscular & 40 & $80 \%$ \\
\hline Posición Prono & 34 & $68 \%$ \\
\hline ECMO & 4 & $8,0 \%$ \\
\hline
\end{tabular}

Los promedios se informan con desviación estándar $( \pm)$. Abreviaturas: N, número de pacientes; DE desviación estándar; PaFi, relación entre la presión parcial de oxígeno y la fracción inspirada de oxígeno; $\mathrm{pCO}_{2}$, presión parcial de dióxido de carbono; ECMO, terapia de oxigenación por membrana extracorpórea.

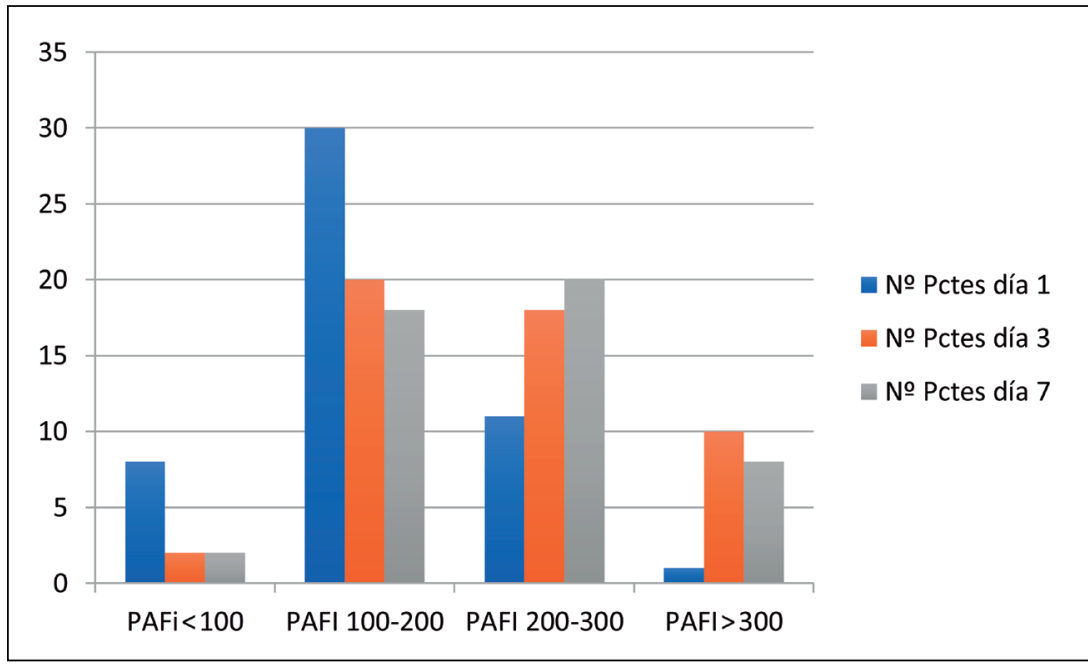

Figura 2. Distribución de los pacientes según la evolución de la PaFi. Distribución de los pacientes según PaFi durante los días 1, 3 y 7 de su estadía en $\mathrm{UCl}$. Se consideraron los intervalos de PaFl definidos por la clasificación de Berlín sobre síndrome de distrés respiratorio agudo. Abreviaturas: PaFi, relación de presión parcial de oxígeno y fracción inspirada de oxígeno; $\mathrm{UCl}$, unidad de cuidados intensivos; No, número; Pctes, pacientes. 
Tabla 3. Exámenes de laboratorio e imágenes en UCI

\begin{tabular}{|c|c|c|}
\hline Variables & $\begin{array}{c}\text { n/ } \\
\text { Mediana }\end{array}$ & $\begin{array}{c}\text { Porcentaje/ } \\
\text { RI }\end{array}$ \\
\hline $\begin{array}{l}\text { Leucocitos/uL } \\
-5.000-10.000 \\
-10.000-15.000 \\
->15.000\end{array}$ & $\begin{array}{l}10 \\
18 \\
22\end{array}$ & $\begin{array}{l}20 \% \\
36 \% \\
44 \%\end{array}$ \\
\hline $\begin{array}{l}\text { Recuento absoluto de linfocitos } \\
-<1.200 \\
->1.200\end{array}$ & $\begin{array}{c}44 \\
6\end{array}$ & $\begin{array}{l}88 \% \\
12 \%\end{array}$ \\
\hline $\begin{array}{l}\text { Plaquetas/uL } \\
-<150.000 \\
-150.000-450.000 \\
->450.000\end{array}$ & $\begin{array}{c}2 \\
32 \\
16\end{array}$ & $\begin{array}{l}4 \% \\
64 \% \\
32 \%\end{array}$ \\
\hline $\begin{array}{l}\text { Lactato } \mathrm{mmol} / \mathrm{L} \\
\text { - Mediana } \\
\text { - } \%>2,2\end{array}$ & $\begin{array}{c}2,22 \\
25\end{array}$ & $\begin{array}{c}1,18-3,07 \\
50 \%\end{array}$ \\
\hline Dímero D ng/mL & 2.295 & $\begin{array}{l}1.112,5- \\
3.802,5\end{array}$ \\
\hline PCR mg/L & 262,5 & $207-369,5$ \\
\hline $\begin{array}{l}\text { LDH U/L } \\
- \text { Mediana } \\
-<220 \\
-220-440 \\
->440\end{array}$ & $\begin{array}{c}665,5 \\
0 \\
8 \\
42\end{array}$ & $\begin{array}{c}519,5-872,8 \\
0 \% \\
16 \% \\
84 \%\end{array}$ \\
\hline $\begin{array}{l}\text { Creatinina } \mathrm{mg} / \mathrm{dL} \\
\text { - Mediana } \\
\text { - }>1,2\end{array}$ & $\begin{array}{c}1,18 \\
22\end{array}$ & $\begin{array}{c}0,92-2,12 \\
44 \%\end{array}$ \\
\hline $\begin{array}{l}\text { TC de tórax } \\
\text { - Típico } \\
\text { - Indeterminado } \\
\text { - Atípico }\end{array}$ & $\begin{array}{c}47 \\
0 \\
3\end{array}$ & $\begin{array}{l}94 \% \\
0 \% \\
6 \%\end{array}$ \\
\hline
\end{tabular}

Las medianas se informan con rango intercuartil. Abreviaturas: $\mathrm{N}$, número de pacientes; $\mathrm{RI}$, rango intercuartil; $\mathrm{PCR}$, proteína $C$ reactiva, $L D H$, lactato deshidrogenasa; TC, tomografía computada. Los resultados de los exámenes de laboratorio fueron determinados considerando todos los exámenes tomados durante los 7 primeros días en $\mathrm{UCl}$.

tuvieron una TC o angiotac de tórax, encontrando en $94 \%$ un patrón típico de COVID-19. Se solicitó un angiotac a 21 pacientes por sospecha de tromboembolismo pulmonar, los que resultaron positivos en 2 casos.

La mayoría de los pacientes recibieron antibióticos e hidroxicloroquina, y un poco más de la mitad corticoides. Ningún paciente presentó alteraciones al electrocardiograma o arritmias. En 17\% de los pacientes se objetivaron infeccio- nes comunitarias concomitantes al COVID-19, principalmente neumonias y bacteriemias por cocáceas gram positivas. Por otro lado, se realizó a 24 pacientes, al ingreso a UCI, un panel molecular de muestras respiratorias, sin encontrar bacterias atípicas. Además de los antibióticos iniciales, $52 \%$ de los pacientes necesitaron antibióticos adicionales por infecciones nosocomiales, siendo la neumonía asociada a ventilación mecánica por bacilos gram negativos, principalmente Klebsiella pneumoniae y Pseudomona aeruginosa, el diagnóstico más frecuente. Los corticoides más utilizados fueron metilprednisolona e hidrocortisona, en equivalentes a 1-2 mg/kg de peso de prednisona al día. La Tabla 4 describe los tratamientos recibidos.

Tabla 4. Tratamientos en $\mathrm{UCl}$ y desenlaces clínicos

\begin{tabular}{|c|c|c|}
\hline & $\begin{array}{c}\text { n/ } \\
\text { Promedio/ } \\
\text { mediana }\end{array}$ & $\begin{array}{c}\text { Porcentaje/ } \\
\text { RI }\end{array}$ \\
\hline Betalactámicos ${ }^{(a)}$ & 43 & $86 \%$ \\
\hline Azitromicina & 40 & $80 \%$ \\
\hline Tocilizumab & 1 & $2 \%$ \\
\hline Hidroxicloroquina & 44 & $88 \%$ \\
\hline Lopinavir/Ritonavir & 21 & $42 \%$ \\
\hline Corticoides $^{(b)}$ & 26 & $52 \%$ \\
\hline $\begin{array}{l}\text { Días de estadía en UCI(c) } \\
\text { - Mediana } \\
\text { - < } 8 \text { días } \\
\text { - 8-14 días } \\
\text { - 15-21 días } \\
\text { - > } 21 \text { días }\end{array}$ & $\begin{array}{c}16 \\
5 \\
16 \\
17 \\
12\end{array}$ & $\begin{array}{c}13-21 \\
10 \% \\
32 \% \\
34 \% \\
24 \%\end{array}$ \\
\hline $\begin{array}{l}\text { Días de conexión a VMI(c) } \\
\text { - Mediana } \\
\text { - < } 8 \text { días } \\
\text { - 8-14 días } \\
\text { - 15-21 días } \\
\text { - > } 21 \text { días }\end{array}$ & $\begin{array}{c}13,5 \\
7 \\
22 \\
12 \\
9\end{array}$ & $\begin{array}{c}9-20 \\
14 \% \\
44 \% \\
24 \% \\
18 \%\end{array}$ \\
\hline Letalidad $^{(c)}$ & 8 & $16 \%$ \\
\hline
\end{tabular}

Las medianas se informan con rango intercuartil. Abreviaturas: N, número de pacientes; RI, rango intercuartil; $\mathrm{UCl}$, unidad cuidados intensivos; VMI, ventilación mecánica invasiva; (a)Los betalactámicos utilizados fueron Ceftriaxona y Ampicilina/Sulbactam; (b)Los corticoides más utilizados fueron dexametasona, metilprednisolona e hidrocortisona; (c)Desenlace clínico determinado en al término del estudio, es decir el día 2 de junio de 2020. Al término del estudio, ningún paciente que hubiera egresado de $\mathrm{UCl}$ había fallecido en otro servicio, correspondiendo todos las muertes a la estadía en UCl. 
Al cierre del seguimiento, que según lo planificado ocurrió 7 días después de enrolar al último paciente, es decir el 2 de junio, había aún 9 pacientes hospitalizados en UCI en VMI. La Tabla 4 muestra la información sobre los días de UCI, días de VMI y letalidad. De los 8 pacientes fallecidos durante el período de seguimiento, todos murieron en UCI (letalidad 16\%). Dos pacientes fallecieron durante la primera semana en esta unidad. El primero murió por un cuadro sugerente de tormenta de citoquinas secundaria a COVID-19. El segundo falleció por una complicación mecánica de un infarto cardíaco. Las otras 6 muertes se produjeron después de la segunda semana en UCI, destacando 4 pacientes que fallecieron por un shock séptico secundario a infecciones sobreagregadas.

\section{Discusión}

Dentro de los resultados de este estudio, destaca que los pacientes eran más jovenes y que hubo un mayor porcentaje de mujeres, respecto a lo mostrado por publicaciones internacionales ${ }^{3}$. En relación a la información nacional, los pacientes de esta cohorte eran más jóvenes que lo reportado en el informe epidemiológico del MINSAL de fines de junio ${ }^{1}$, con un porcentaje de pacientes menores de 60 años de $68 \%$ versus $50 \%$ respectivamente. Otras características son similares a lo descrito por otros autores, como el alto porcetaje de obesidad y sobrepeso ${ }^{5,12}$; esto se puede explicar por la elevada prevalencia de estas condiciones en Chile ${ }^{13}$. En relación con los días transcurridos desde el inicio de los síntomas hasta la conexión a VMI, el intervalo más frecuente fue de 5-8 días, lo que coincide con la fase inflamatoria del COVID-1914,15.

La estadía en VMI y UCI fueron mayores a las encontradas previamente en nuestra unidad en una población mixta de pacientes $\operatorname{críticos}^{16}$. Otros autores también han reportado un aumento de los días de UCI y VMI por esta patología $^{15,17}$. Ante estas características del COVID-19, Marini y Gattinoni han señalado que causa una injuría pulmonar única, denominándola CARDS (COVID-19 Acute Respiratory Distress Syndome $)^{18}$.

Los niveles de PEEP utilizados en esta cohorte fueron menores a los publicados por otros estudios ${ }^{3}$. Esto podría explicarse porque el seguimiento de la VMI consideró los primeros 7 días, lo que probablemente coincidió con la fase precoz del CARDS. Según Gattinoni, durante los primeros días del compromiso pulmonar por COVID-19 los pacientes tendrían una alta compliance pulmonar y un escaso potencial de reclutamiento, lo que este autor ha reconocido como fenotipo "L"19. Otra característica que llama la atención de este estudio es el importante uso de prono. Se ha propuesto que en las etapas iniciales del CARDS el prono sería beneficioso en mejorar la oxigenación al facilitar la redistribución de la perfusión pulmonar por efecto de fuerzas gravitacionales ${ }^{20}$.

En relación a los exámenes de laboratorio, se encontró una alta prevalencia de linfopenia, asociada a elevación de DD, LDH y PCR. Estas variables se han reconocido como marcadores pronósticos en COVID-1921. Dada la naturaleza descriptiva de este estudio, no se analizó si había alguna relación entre estos resultados y la evolución de los pacientes.

Estos pacientes presentaron otras disfunciones, además de la respiratoria, lo que se traduce en el SOFA de ingreso a UCI. Casi la mitad de los pacientes presentaron alteraciones de la función renal, lo que coincide con otras publicaciones ${ }^{15,22}$, siendo la segunda disfunción más frecuente. La letalidad fue similar a la reportada previamente por nuestro grupo ${ }^{16}$ y menor a la consignada en otros estudios sobre COVID-195,17. Aunque esta letalidad podría modificarse con una muestra mayor o con un segumiento más prolongado, es interesante la disociación encontrada en esta cohorte entre morbilidad (mayor estadía en UCI y VMI que la habitual en nuestra unidad) y letalidad. Esta disociación también está presente en otros reportes ${ }^{2}$ y por supuesto se requieren más estudios al respecto.

Como se señaló previamente, los pacientes de esta cohorte fueron manejados de acuerdo con el protocolo farmacológico de COVID-19 del CASR ${ }^{7}$ del 7 de abril, el que se basaba en las recomendaciones nacionales e internacionales ${ }^{23,24}$ generadas durante los primeros meses de la pandemia. Esto implicó un alto uso de hidroxicloroquina y un moderado uso de lopinavir/ ritonavir en los pacientes de este estudio. Ya que la evidencia sobre los efectos de estos medicamentos en COVID-19 es contradictoria ${ }^{25-28}$, posterior al cierre del seguimiento de este estudio, 
estos medicamentos fueron retirados del protocolo institucional, según consta en una versión actualizada de dicha guía de fines de mayo ${ }^{29}$. Por otro lado, el uso de corticoides ha ganado importancia en el último tiempo, con evidencia de mejoría en mortalidad ${ }^{30,31}$, estableciéndose como pilar del manejo de los pacientes graves con COVID-19. Es esperable que en futuros reportes de pacientes críticos de nuestro centro aumente el uso corticoídes en COVID-19. En relación con el Tociluzumab, fármaco que bloquea la cadena inflamatoria mediada por interleucina $6^{32}$, la evidencia sobre su efecto en COVID-19 proviene de series pequeñas ${ }^{33-35}$; en el presente estudio su uso fue mínimo, lo que no permite establecer conclusiones.

En este estudio se encontró una alta incidencia de coinfección bacteriana, lo que también ha sido reportado en otras publicaciones. Zhu mostró una prevalencia de 91,8\% de coinfección bacteriana en 257 muestras respiratorias de pacientes con COVID-19 ${ }^{36}$.

Este estudio tiene varias limitaciones, como que al cierre del seguimiento aún había pacientes en UCI, lo que impacta en variables como los días de UCI y VMI. Esta también ha sido una limitación de otros estudios sobre COVID-19 que han incluido pacientes críticos ${ }^{5}$. Otra limitación, es que algunos de los médicamentos utilizados en estos primeros 50 pacientes ya no forman parte del protocolo de manejo de COVID-19 del $\mathrm{CASR}^{29}$. Sin embargo, dado lo dinámico que es la generación de conocimientos en una enfermedad nueva como esta, es esperable que los tratamientos evolucionen rápidamente. Por último, en este primer reporte de pacientes críticos con COVID-19 del CASR, se consideraron desenlaces de UCI. En futuros estudios sería ideal considerar resultados a mediano plazo, como la funcionalidad de los pacientes posterior al CARDS.

La información obtenida en este estudio caracteriza de manera preliminar a los pacientes con requerimientos de VMI por COVID-19 admitidos en la UCI del CASR. Aunque este estudio tiene limitaciones, consideramos que es importante contar con datos locales de esta pandemia, que sin duda ha sido el mayor desafío que la medicina intensiva ha debido enfrentar. Estos datos permitirán mejorar el manejo de los pacientes con COVID-19 en nuestro centro y podrían ser un aporte para otras UCIs en nuestro país.

\section{Referencias}

1. Ministerio de Salud de Chile (MINSAL). Informe Epidemiológico No 28, Enfermedad por SARS-COV-2 (COVID-19). Chile 23/06/2020. Departamento de Epidemiología. Disponible en: https://www.minsal.cl/ wp-content/uploads/2020/06/16062020_Reporte_Covid19.pdf. [Consultado el 28 de junio de 2020].

2. Wang D, Hu B, Hu C, Zhu F, Liu X, Zhang J, et al. Clinical Characteristics of 138 Hospitalized Patients With 2019 Novel Coronavirus-Infected Pneumonia in Wuhan, China. JAMA 2020; 323 (11): 1061-9.

3. Grasselli G, Zangrillo A, Zanella A, Antonelli M, Cabrini L, Castelli A, et al. Baseline Characteristics and Outcomes of 1591 patients Infected With SARS-CoV-2 Admitted to ICUs of the Lombardy Region, Italy. JAMA 2020; 323 (16): 1574-81.

4. Guan WJ, Ni ZY, Hu Y, Liang WH, Ou CQ, He JX, et al. Clinical Characteristics of Coronavirus Disease 2019 in China. N Engl J Med 2020; 382 (18): 1708-20.

5. Richardson S, Hirsch J, Narasimhan M, Crawford J, McGinn T, Davidson K, et al. Presenting Characteristics, Comorbidities, and Outcomes Among 5700 Patients Hospitalized With COVID-19 in the NewYork City Area. JAMA 2020; 323 (20): 2052-9.

6. Ministerio de Salud de Chile (MINSAL), unidad de estadística Servicio de Salud Metropolitano Sur Oriente. Proyección de población INE en base a censo 2017, por comuna, sexo y grupo etario Servicio de salud Metropolitano Sur Oriente período: 2018-2035. Disponible en http://estadistica.ssmso.cl/poblacion-ssmso/ [Consultado el 19 de junio de 2020].

7. Ministerio de Salud de Chile (MINSAL), Servicio de Salud Metropolitano Sur Oriente. Protocolo tratamiento farmacológico para pacientes con COVID-19, 07/04/2020. Disponible en: https://covid.ssmso.cl/. [Consultado el 19 de junio de 2020].

8. Metlay JP, Waterer GW, Long AC, et al. Diagnosis and Treatment of Adults with Community-acquired Pneumonia. An Official Clinical Practice Guideline of the American Thoracic Society and Infectious Diseases Society of America. Am J Respir Crit Care Med 2019; 200 (7): e45-e67.

9. Guérin C, Reignier J, Richard JC, Beuret P, Gacouin A, Boulain T, et al. Prone positioning in severe acute respiratory distress syndrome. N Engl J Med 2013; 368 (23): 2159-68.

10. Simpson S, Kay FU, Abbara S, Bhalla S, Chung J, Chung M, et al. Radiological Society of North America Expert Consensus Statement on Reporting Chest CT Findings Related to COVID-19. Endorsed by the Society of Tho- 
racic Radiology, the American College of Radiology, and RSNA. J Thorac Imaging 2020; 35 (4): 219-27.

11. ARDS Definition Task Force. Acute Respiratory Distress Syndrome: The Berlin Definition. JAMA 2012; 308 (23): 2526-33.

12. Lemyze M, Courageux N, Maladobry T, Arumadura C, Pauquet P, Orfi A, et al. Implications of Obesity for the Management of Severe Coronavirus Disease 2019 Pneumonia. Crit Care Med 2020, en prensa. Disponible en: https://europepmc.org/article/pmc/pmc7255399. [Consultado el 15 de junio de 2020].

13. Ministerio de salud de Chile (MINSAL). Encuesta Nacional de Salud 2016-2017. Disponoble en: http://epi. minsal.cl/wp-content/uploads/2018/03/1\%C2\%BA-Resultados ENS_DEPTO.EPIDEMIOLOGIA.MINSAL.pdf. [Consultado el 15 de junio de 2020].

14. Siddiqi H, Mehra M. COVID-19 illness in native and immunosuppressed states: A clinical-therapeutic staging proposal. J Heart Lung Transplant 2020; 39(5): 405-7.

15. Bhatraju P, Ghassemieh B, Nichols M, Kim R, Jerome $\mathrm{K}$, Nalla A, et al. Covid-19 in Critically Ill Patients in the Seattle Region - Case Series. N Engl J Med 2020; 382 (21): 2012-22.

16. Ruiz C, Díaz MA, Zapata JM, Bravo S, Panay S, Escobar C, et al. Características y evolución de los pacientes que ingresan a una Unidad de Cuidados Intensivos de un hospital público. Rev Med Chile 2016; 144: 1297-304.

17. Zangrillo A, Beretta L, Scandroglio AM, Mont Gi, Fominskiy E, Colombo S, et al. Characteristics, treatment, outcomes and cause of death of invasively ventilated patients with COVID-19 ARDS in Milan, Italy. Crit Care Resusc 2020, en prensa. Disponible en: https://pubmed. ncbi.nlm.nih.gov/32353223/. [Consultado el 15 de junio de 2020].

18. Gattinini L, Marini J. Management of COVID-19 Respiratory Distress. JAMA 2020; 323 (22): 2329-30.

19. Gattinoni L, Chiumello D, Caironi P, Busana M, Romitti F, Brazzi L, et al. COVID-19 pneumonia: different respiratory treatments for different phenotypes. Intensive Care Med 2020; 46 (6): 1099-1102.

20. Gattinoni L, Coppola S, Cressoni M, Busana M, Rossi S, Chiumello D. Covid-19 Does Not Lead to a "Typical" Acute Respiratory Distress Syndrome. Am J Respir Crit Care Med 2020; 201 (10): 1299-300.

21. Wu C, Chen X, Cai Y, Xia J, Zhou X, Xu S, et al. Risk Factors Associated With Acute Respiratory Distress Syndrome and Death in Patients With Coronavirus Disease 2019 Pneumonia in Wuhan, China. JAMA 2020, en prensa. Disponible en: https://jamanetwork. com/journals/jamainternalmedicine/fullarticle/2763184. [Consultado el 19 de junio de 2020].
22. Hirsch J, Ng J, Ross D, Sharma P, Shah H, BarnettS RL, et al. [Acute kidney injury in patients hospitalized with COVID-19. Kidney Int 2020, en prensa. Disponible en: https://www.kidney-international.org/article/S00852538(20)30532-9/pdf. [Consultado el 15 de junio de 2020].

23. Alhazzani W, Møller MH, Arabi YM, Loeb M, Gong M, Fan E, et al. Surviving Sepsis Campaign: guidelines on the management of critically ill adults with Coronavirus Disease 2019 (COVID-19)]. Intensive Care Med 2020; 46 (5): 854-87.

24. Bhimraj A, Morgan RL, Shumaker AH, Lavergne V, Baden L, Chi-Chung Cheng V, et al. Infectious Diseases Society of America Guidelines on the Treatment and Management of Patients with COVID-19. Clin Infect Dis 2020, en prensa. Disponible en: https://pubmed. ncbi.nlm.nih.gov/32338708/. [Consultado el 19 de junio del 2020].

25. Tang W, Cao Z, Han M, Wang Z, Chen J, Sun W, et al. Hydroxychloroquine in patients with mainly mild to moderate coronavirus disease 2019: open label, randomised controlled trial. BMJ 2020;369:m1849. Disponible en: https://www.bmj.com/content/369/bmj.m1849.long. [Consultado el 19 de junio de 2020].

26. Mercuro NJ, Yen CF, Shim DJ, Maher TR, McCoy CM, Zimetbaum PJ, et al. Risk of QT Interval Prolongation Associated With Use of Hydroxychloroquine With or Without Concomitant Azithromycin Among Hospitalized Patients Testing Positive for Coronavirus Disease 2019 (COVID-19). JAMA Cardiol 2020; 201834. Disponible en: https://jamanetwork.com/journals/jamacardiology/fullarticle/2765631. [Consultado el 19 de junio de 2020].

27. Cao B, Wang Y, Wen D, Liu W, Wang J, Fan G, et al. A Trial of Lopinavir-Ritonavir in Adults Hospitalized with Severe Covid-19. N Engl J Med 2020; 382 (19): 1787-99.

28. Borba MGS, Val FFA, Sampaio VS, Araújo M, Cardoso G, Brito M, et al. Effect of High vs Low Doses of Chloroquine Diphosphate as Adjunctive Therapy for Patients Hospitalized With Severe Acute Respiratory Syndrome Coronavirus 2 (SARS-CoV-2) Infection: A Randomized Clinical Trial. JAMA Netw Open 2020; 3 (4): e208857. Disponible en: https:/es.jamanetwork.com/journals/ jamanetworkopen/fullarticle/2765499. [Consultado el 19 de junio de 2020].

29. Ministerio de Salud de Chile (MINSAL), Servicio de Salud Metropolitano Sur Oriente. Protocolo tratamiento farmacológico para pacientes con COVID-19, 24/05/2020. Disponible en: https://covid.ssmso.cl/ . [Consultado el 19 de junio de 2020]. 
30. Fadel R, Morrison A, Vahia A, Smith Z, Chaudhry Z, Bhargava P, et al. [Early Short Course Corticosteroids in Hospitalized Patients with COVID-19]. JAMA Intern Med 2020 Mar 13. Disponible en: https://pubmed.ncbi. nlm.nih.gov/32427279/. [Consultado el 19 de junio de 2020].

31. Horby P, Landray M on behaft of RECOVERY Collaborative Group. Effect of Dexamethasone in Hospitalized Patients with COVID-19-Preliminary Report. Disponible en: https://www.recoverytrial.net/files/hcq-recovery-statement-050620-final-002.pdf. [Consultado el 19 de junio de 2020].

32. Tay MZ, Poh CM, Rénia L, MacAry P, Ng L. The trinity of COVID-19: immunity, inflammation and intervention. Nat Rev Immunol 2020; 20 (6): 363-74. [Consultado el 19 de junio de 2020].

33. Sciascia S, Aprà F, Baffa A, Baldovino S, Boaro D, Boero $\mathrm{R}$, et al. Pilot prospective open, single-arm multicentre study on off-label use of tocilizumab in patients with severe COVID-19. Clin Exp Rheumatol 2020; 38 (3):
529-32.

34. Klopfenstein T, Zayet S, Lohse A, Balblanc JC, Badie J, Royer PY, et al. Tocilizumab therapy reduced intensive care unit admissions and/or mortality in COVID-19 patients. Med Mal Infect 2020, en prensa. Disponible en:https://www.sciencedirect.com/science/article/pii/ S0399077X20301293?via\%3Dihub. [Consultado el 19 de junio de 2020].

35. Campochiaro C, Della-Torre E, Cavalli G, De Luca G, Ripa M, Boffini, N, et al. Efficacy and safety of tocilizumab in severe COVID-19 patients: a single-centre retrospective cohort study. Eur J Intern Med 2020; 76: 43-9. Disponible en: https://www.ncbi.nlm.nih.gov/ pmc/articles/PMC7242960/. [Consultado el 19 de junio de 2020].

36. Zhu X, Ge Y, Wu T, Zhao K, Chen Y, Wu B, et al. Co-infection with respiratory pathogens among COVID-2019 cases. Virus Res 2020; 285: 198005. Disponible en: https://www.ncbi.nlm.nih.gov/pmc/articles/ PMC7213959/. [Consultado el 19 de junio de 2020]. 